\title{
Insulin Resistance Is Associated with Early Gastric Cancer: A Prospective Multicenter Case Control Study
}

Hye Jung Kwon ${ }^{1}$, Moo In Park ${ }^{1}$, Seun Ja Park ${ }^{1}$, Won Moon ${ }^{1}$, Sung Eun Kim ${ }^{1}$, Jae Hyun Kim ${ }^{1}$, Youn Jung Choi ${ }^{1}$, and Sang Kil Lee ${ }^{2}$

${ }^{1}$ Division of Gastroenterology, Department of Internal Medicine, Kosin University College of Medicine, Busan, and ${ }^{2}$ Division of Gastroenterology, Department of Internal Medicine, Institute of Gastroenterology, Severance Hospital, Yonsei University College of Medicine, Seoul, Korea

See editorial on page 133.

Background/Aims: Recently, increased body weight has been found to be associated with an increasing risk of several cancers, including gastric cancer. The true pathogenic role of hyperglycemia in the development of gastric cancer remains unclear as hyperglycemia and its associated conditions may work as carcinogenic factors. The goal of this study was to clarify the factors associated with early gastric cancer and evaluate a homeostasis model assessment of the insulin resistance (HOMA-IR) index, fasting glucose, and lipid profile as predictors of early gastric cancer. Methods: A total of 63 patients with early gastric cancer between November 2012 and March 2013 were included. Preoperative serum lipid profile levels and serum fasting glucose were examined prospectively in patients with early gastric cancer. The same number of controls were evaluated and matched to the early gastric cancer group for age and gender. We performed multivariate logistic regression analysis to identify independent risk factors for early gastric cancer. Results: Univariate analysis showed that risk for early gastric cancer was associated with diastolic blood pressure (BP), total cholesterol, fasting glucose, and HOMA-IR. In the multivariate-adjusted model, higher total cholesterol, fasting glucose, body mass index, and diastolic BP were strongly associated with an increased risk of early gastric cancer. Conclusions: Hyperglycemia, a lower high-density lipoprotein cholesterol level, and a low HOMA-IR level appear to be associated with early gastric cancer risk. (Gut Liver 2019;13:154-160)

Key Words: Early gastric cancer; Hyperglycemia; HOMA-IR; Adiponectin; Cholesterol

\section{INTRODUCTION}

Gastric cancer is one of the most common causes of cancer induced deaths. Because of the survival of patients that have advanced gastric cancer is almost poor, early detection, and proper resection of the gastric malignant lesion at an earlier stage improves the survival. ${ }^{1-3}$ More effective approach for surveillance of early gastric cancer (EGC) is needed. Various studies have improved that Helicobacter pylori infection is obvious risk factor for the carcinogenesis of stomach. ${ }^{4}$ However in addition to infection of $H$. pylori, multiple cofactors would play a role in gastric carcinogenesis. There were many evidences prove that patients of diabetes mellitus (DM) had a higher risk of cardiovascular disease and malignancies in various organs. ${ }^{5-8} \mathrm{Nev}-$ ertheless, few studies had focused on the relationship between DM, hyperglycemic state and the development of EGC. ${ }^{9}$ Authors have a question as to whether the development of EGC is related to insulin resistant state, not overt DM.

Recently, there has been a study that adiponectin, one kind of adipokine, was elevated in advanced gastric cancer. ${ }^{10,11}$ The adiponectin is associated with the pathogenesis of DM through the regulation of glucose, metabolism of fatty acid, and insulin sensitivity in various epithelial and stromal cells. ${ }^{12,13}$ We measured it in a Korean population and examined the relationship between the development of EGC and plasma adiponectin levels.

In this study, we evaluated insulin resistance (fasting glucose, fasting insulin, glycated hemoglobin [HbA1c], homeostasis model assessment of the insulin resistance [HOMA-IR], and HOMA $\beta$-cell function), lipid profile (total cholesterol [TC], highdensity lipoprotein [HDL], low-density lipoprotein [LDL], and triglyceride [TG]) and plasma level of adiponectin in EGC patients and examined a possible role for adipokines in the development

Correspondence to: Moo In Park (https://orcid.org/0000-0003-2071-6957)

Division of Gastroenterology, Department of Internal Medicine, Kosin University College of Medicine, 262 Gamcheon-ro, Seo-gu, Busan 49267, Korea

Tel: +82-51-990-6103, Fax: +82-51-990-5055, E-mail: mipark@ns.kosinmed.or.kr

Received on December 11, 2017. Revised on August 8, 2018. Accepted on August 8, 2018. Published online January 3, 2019

pISSN 1976-2283 eISSN 2005-1212 https://doi.org/10.5009/gnl17556

(a) This is an Open Access article distributed under the terms of the Creative Commons Attribution Non-Commercial License (http://creativecommons.org/licenses/by-nc/4.0) which permits unrestricted non-commercial use, distribution, and reproduction in any medium, provided the original work is properly cited. 
of EGC.

\section{MATERIALS AND METHODS}

\section{Patients}

This study is a case control prospective study performed in two centers (Kosin University Gospel Hospital and Yonsei Severance Hospital), from October 1, 2012 to March 31, 2013. We performed sample size assessment and determined that a total of 194 participants were needed, 97 in each group (EGC and controls). The inclusion criteria were aged between 18 to 75 years old with written informed consent. The EGC group included patients who underwent endoscopic submucosal dissection or gastrectomy with conventional lymph node dissection and received pathological diagnosis of EGC. The control group was matched for age and sex (1:1) to the EGC group and subjects were enrolled from the health promotion center of each hospital. Subjects in both groups were excluded if they had any active malignancies (except gastric cancer for the EGC group) or a past history of any other malignancies. Exclusion criteria were patients with peptic ulcer disease or gastroesophageal reflux disease, oral hypoglycemic agents or insulin users due to diabetes, and patients taking oral lipid lowering agents due to hypercholesterolemia. Study protocol was reviewed and approved by the Institutional Review Board (IRB No. KAIRB 13-152).

\section{Assessment of study protocol}

Clinical and pathological information of all subjects were obtained from medical records. The body mass index (BMI) at the time of diagnosis was calculated based on weight $(\mathrm{kg}) /$ height $(\mathrm{m})^{2}$. Subjects (EGC and control group) were provide informed consent prior to the collection and analysis of blood samples.
Serum was collected from all subjects after overnight or 12 hours of fasting time. We requested that each subject be provided with a $10 \mathrm{~mL}$ blood sample at the time of admission or health checkup. The collected blood samples were stored at $-80^{\circ} \mathrm{C}$ until analysis.

Several parameters were measured that fasting glucose, $\mathrm{HbA1c}$, and fasting insulin. We also investigated HOMA-IR and HOMA $\beta$-cell function as a value reflecting insulin resistance. The HOMA-IR score was calculated by the formula: (fasting insulin $[\mu \mathrm{U} / \mathrm{mL}] \times$ fasting glucose $[\mathrm{mmol} / \mathrm{L}]) / 22.5$. HOMA-IR $\geq 1.96$ was defined as the presence of insulin resistance ${ }^{5}$ HOMA $\beta$-cell function was calculated by the formula: (20xfasting insulin $[\mu \mathrm{U} /$ $\mathrm{mL}]$ )/(fasting glucose [mmol]-3.5). ${ }^{5,14}$ Serum TC, TG, HDL and LDL values were determined by enzymatic methods.

Plasma adiponectin levels were measured using an adiponectin ELISA kit (Otsuka Pharmaceutical Co. Ltd., Tokushima, Japan). The adiponectin concentration was divided into two classes: The level of Adiponectin $\geq 8.0 \mathrm{ng} / \mathrm{dL}$ and $<8.0 \mathrm{ng} / \mathrm{dL} .{ }^{15}$ $H$. pylori infection status was considered positive if the subjects had either $H$. pylori antibody $\geq 10 \mathrm{U} / \mathrm{mL}^{15}$

\section{Statistical analysis}

For continuous variables, the results expressed as mean \pm standard deviation or median. The Student t-test and chi-square test were used to compare basic characteristics between the EGC and control groups. Matched odds ratios (ORs) and their corresponding 95\% confidence intervals (CIs) were calculated using logistic regression models. p-values less than 0.05 were defined as statistically significant. All statistical analyses were performed with SPSS version 8.0 (SPSS Inc., Chicago, IL, USA).

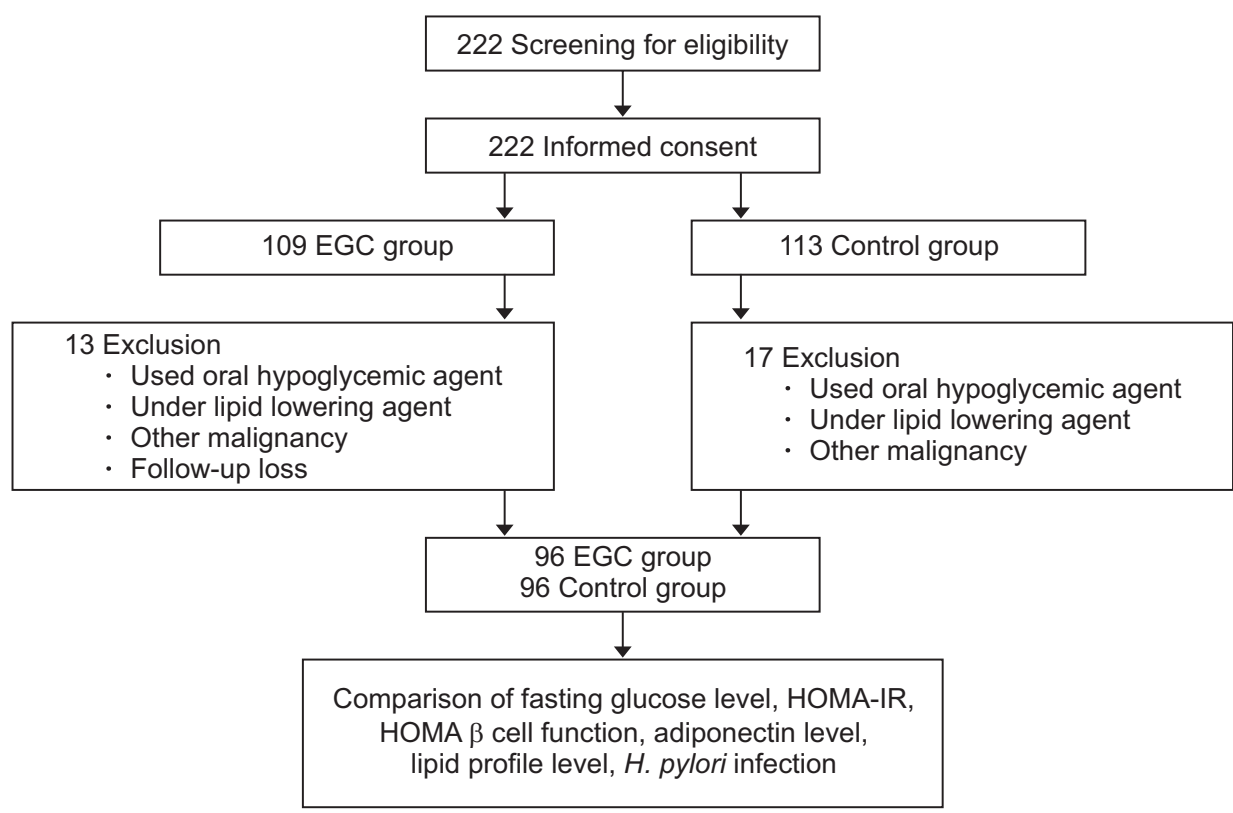

Fig. 1. Study flowchart. EGC, early gastric cancer; HOMAIR, homeostasis model assessment of the insulin resistance; $H$. pylori; Helicobacter pylori. 


\section{RESULTS}

From November 2012 to March 2013, we initially recruited a total of 222 participants. Among them, 13 participants in the EGC group were excluded because of use of oral hypoglycemic agents or lipid lowering agents or the presence of other malignant disease. An additional 17 participants were excluded from the control group because of the presence of another malignant disease. We therefore included 96 individuals in the EGC group and control group, respectively, according to inclusion criteria. The study flow showed Fig. 1. Each group included 66 men and 30 women. Table 1 shows the basic characteristics of the two groups. There was no difference in height, weight, and BMI between the two groups, and the mean age was 55.6 years. The rate of $H$. pylori infection was higher in the EGC group than the control group. There were no statistically significant differences in the rate of alcohol consumption and smoking between groups. Fasting glucose, fasting insulin, HOMA-IR, HbA1c, TC, HDL, and diastolic blood pressure were statistically significant in the EGC group compared with control group. Although this difference was not statistically significant, serum level of adiponectin was lower in the EGC group (Table 1). The univariate analyses showed that risk of cancer was slightly increased

Table 1. Baseline Patient Characteristics of Early Gastric Cancer and Control Groups

\begin{tabular}{|c|c|c|c|}
\hline Characteristic & $\begin{array}{c}\text { EGC } \\
(n=96)\end{array}$ & $\begin{array}{c}\text { Control } \\
(n=96)\end{array}$ & p-value \\
\hline Age, yr & $55.6 \pm 11.73$ & $55.6 \pm 11.73$ & 0.021 \\
\hline Sex, male/female & $66 / 30$ & $66 / 30$ & \\
\hline BMI, $\mathrm{kg} / \mathrm{m}^{2}$ & $23.80 \pm 2.90$ & $24.30 \pm 2.42$ & 0.195 \\
\hline $\mathrm{SBP}, \mathrm{mm} \mathrm{Hg}$ & $124.7 \pm 12.80$ & $123.6 \pm 9.87$ & 0.500 \\
\hline $\mathrm{DBP}, \mathrm{mm} \mathrm{Hg}$ & $79.08 \pm 7.67$ & $75.42 \pm 7.39$ & 0.009 \\
\hline $\mathrm{AC}, \mathrm{cm}$ & $81.77 \pm 7.57$ & $83.08 \pm 8.069$ & 0.297 \\
\hline Total cholesterol, mg/dL & $179.3 \pm 35.47$ & $196.2 \pm 35.61$ & 0.001 \\
\hline $\mathrm{HDL}, \mathrm{mg} / \mathrm{dL}$ & $46.83 \pm 11.46$ & $51.48 \pm 12.28$ & 0.007 \\
\hline $\mathrm{LDL}, \mathrm{mg} / \mathrm{dL}$ & $104.5 \pm 33.62$ & $112.5 \pm 33.89$ & 0.102 \\
\hline Triglyceride, mg/dL & $129.8 \pm 71.84$ & $139.7 \pm 136.5$ & 0.529 \\
\hline TG/HDL index & $3.09 \pm 2.17$ & $3.26 \pm 5.39$ & 0.773 \\
\hline Fasting glucose, mg/dL & $98.61 \pm 15.89$ & $87.41 \pm 13.30$ & 0.001 \\
\hline Fasting insulin, mg/dL & $6.27 \pm 7.09$ & $4.33 \pm 3.16$ & 0.015 \\
\hline HOMA-IR & $1.56 \pm 1.71$ & $0.96 \pm 0.75$ & 0.002 \\
\hline HOMA $\beta$-cell & $1.30 \pm 0.46$ & $1.28 \pm 0.45$ & 0.752 \\
\hline Adiponectin, ng/mL & $8.28 \pm 4.98$ & $8.61 \pm 6.23$ & 0.685 \\
\hline $\mathrm{HbA} 1 \mathrm{C}, \%$ & $5.82 \pm 0.47$ & $5.63 \pm 0.38$ & 0.002 \\
\hline
\end{tabular}

Data are presented as mean \pm SD or number

EGC, early gastric cancer; BMI, body mass index; SBP, systolic blood pressure; DBP, diastolic blood pressure; AC, abdominal circumference; HDL, high-density lipoprotein; LDL, low-density lipoprotein; TG, triglyceride; HOMA-IR, homeostasis model assessment of the insulin resistance; $\mathrm{HbA} 1 \mathrm{C}$, glycated hemoglobin. among persons with a higher level of fasting glucose, fasting insulin, HOMA-IR, HbA1c, and a lower level of TC and HDL. $H$. pylori infection was a significant risk factor in the EGC group $(\mathrm{p}=0.003)$. BMI, HOMA $\beta$-cell function, LDL, TG level, and adiponectin concentration were not associated with the risk of EGC development in this study (Table 2). The multivariate logistic regression analysis showed that high level of fasting glucose $(\mathrm{OR}=1.08, \mathrm{p}<0.01)$, high level of fasting insulin $(\mathrm{OR}=1.69$, $\mathrm{p}=0.02$ ), HOMA-IR (OR=8.85, $\mathrm{p}=0.03)$, and lower level of TC $(\mathrm{OR}=1.01, \mathrm{p}=0.005)$ were associated with the development of EGC (Table 3). HDL level was not affected in multivariate analysis.

\section{DISCUSSION}

This is prospective, population-based study to report a positive association between insulin resistance and development of EGC in the absence of overt DM. This prospective study provided several salient findings. First, hyperglycemia $(p=0.000$, $\mathrm{OR}=1.066$ ) and insulin resistance (fasting insulin $[\mathrm{p}=0.024$, $O R=1.084]$, HOMA-IR $[\mathrm{p}=0.006, \mathrm{OR}=1.532]$ and $\mathrm{HbA} 1 \mathrm{C}$ $[p=0.004,0 R=3.225])$ were risk factors for the development of EGC. Second, a low level of TC $(p=0.005,0 R=1.015)$ was associated with the development of EGC. Third, although plasma adiponectin level was lower in EGC group, but there was no statistical significance. In a recent study, Ikeda et al. ${ }^{16}$ reported that hyperglycemia (fasting glucose $\geq 5.3 \mathrm{mmol} / \mathrm{L}$ ) increased the risk of gastric cancer induced by $H$. pylori infection. Similar to their data, our prospective case control study demonstrated that the presence of hyperglycemia, hyperinsulinemia and elevated HOMA-IR were significantly related to the development of EGC based on multivariate analyses. A previous study showed that the incidence of gastric cancer is greater in patients with a higher level of $\mathrm{HbA} 1 \mathrm{C},{ }^{17}$ and our data support this relationship $(\mathrm{p}<0.01, \mathrm{OR}=1.08)$. As the previous studies included AGC, it could not rule out secondary hyperglycemia as cancer progress. This study targeted EGC only excluding other variables, which makes higher possibility of EGC acting as main cause. There have been several studies dealing with relevancy between gastric dysplasia and hyperglycemia but this is the first time studying for EGC only. We also investigated plasma fasting insulin, calculated HOMA-IR and HOMA $\beta$-cell function that reflect insulin resistance. ${ }^{5,13}$ Among them, fasting insulin $(\mathrm{p}=0.02$, $\mathrm{OR}=1.69)$ and HOMA-IR $(\mathrm{p}=0.03, \mathrm{OR}=8.85)$ were confirmed to contribute to the development of EGC.

Why does gastric cancer development occur more common in hyperglycemic state than in without hyperglycemia? The exact pathophysiological role of hyperglycemia in the development of stomach cancer remains to be unclear but several possible mechanisms have been suggested. The production of oxygen free radicals that cause DNA damage ${ }^{18}$ increases in hyperglycemic state. High levels of glucose have been shown to contrib- 
Table 2. Univariate Logistic Regression Analysis for the Risk of Early Gastric Cancer

\begin{tabular}{|c|c|c|c|c|}
\hline Variable & EGC $(n=96)$ & Controls $(n=96)$ & OR $(95 \% \mathrm{CI})$ & p-value \\
\hline BMI, $\mathrm{kg} / \mathrm{cm}^{2}$ & & & $0.931(0.837-1.037)$ & 0.1957 \\
\hline$<18.5$ & 4 & 0 & & \\
\hline $18.5-25$ & 66 & 60 & & \\
\hline$\geq 25$ & 26 & 36 & & \\
\hline $\mathrm{FBS}, \mathrm{mg} / \mathrm{dL}$ & & & $1.066(1.037-1.096)$ & 0.0001 \\
\hline$<100$ & 60 & 89 & & \\
\hline$\geq 100$ & 36 & 7 & & \\
\hline Fasting insulin, mg/dL & & & $1.084(1.011-1.162)$ & 0.0243 \\
\hline$<6.20$ & 70 & 73 & & \\
\hline$\geq 6.20$ & 26 & 23 & & \\
\hline HOMA-IR & & & $1.532(1.134-2.071)$ & 0.0055 \\
\hline$<1.96$ & 75 & 85 & & \\
\hline$\geq 1.96$ & 21 & 11 & & \\
\hline HOMA $\beta$-cell function & & & $0.904(0.485-1.685)$ & 0.7508 \\
\hline$<1.18$ & 67 & 69 & & \\
\hline$\geq 1.18$ & 29 & 27 & & \\
\hline $\mathrm{HbA1C}$ & & & $3.225(1.455-7.147)$ & 0.0039 \\
\hline$<6.0$ & 71 & 84 & & \\
\hline$\geq 6.0$ & 25 & 12 & & \\
\hline Adiponectin, ng/dL & & & $0.99(0.941-1.041)$ & 0.6847 \\
\hline$<8.0$ & 53 & 61 & & \\
\hline$\geq 8.0$ & 43 & 35 & & \\
\hline Total cholesterol, mg/dL & & & $0.987(0.978-0.995)$ & 0.0018 \\
\hline$<200$ & 68 & 60 & & \\
\hline $200-239$ & 25 & 25 & & \\
\hline$\geq 240$ & 3 & 11 & & \\
\hline $\mathrm{HDL}, \mathrm{mg} / \mathrm{dL}$ & & & $0.967(0.943-0.992)$ & 0.0089 \\
\hline$<40$ & 30 & 17 & & \\
\hline$\geq 40$ & 63 & 79 & & \\
\hline $\mathrm{LDL}, \mathrm{mg} / \mathrm{dL}$ & & & $0.993(0.984-1.001)$ & 0.1042 \\
\hline$<100$ & 49 & 35 & & \\
\hline $100-129$ & 26 & 37 & & \\
\hline $130-159$ & 17 & 13 & & \\
\hline$\geq 160$ & 4 & 11 & & \\
\hline Triglyceride, $\mathrm{mg} / \mathrm{dL}$ & & & $0.999(0.996-1.002)$ & 0.5363 \\
\hline$<150$ & 73 & 69 & & \\
\hline$\geq 150$ & 23 & 27 & & \\
\hline H. pylori infection & & & - & 0.003 \\
\hline Positive & 76 & 57 & & \\
\hline Negative & 20 & 39 & & \\
\hline
\end{tabular}

EGC, early gastric cancer; OR, odds ratio; CI, confidence interval; BMI, body mass index; FBS, fasting blood sugar; HOMA-IR, homeostasis model assessment of the insulin resistance; HbA1C, glycated hemoglobin; HDL, high-density lipoprotein; LDL, low-density lipoprotein; H. pylori, Helicobacter pylori.

ute to DNA damage in hyperglycemic patients. ${ }^{19}$ To the next, hyperinsulinemia itself is another possible mechanism of the development of gastric malignancy. Insulin is a key regulator of carcinogenesis in several organs including gastric cancer. ${ }^{20-23}$ 
Table 3. Multivariate Logistic Regression Analysis for the Risk of Early Gastric Cancer

\begin{tabular}{lrccc}
\hline \multicolumn{1}{c}{ Variable } & \multicolumn{1}{c}{$\beta$} & SE & p-value & OR (95\% CI) \\
\hline DBP & -0.920 & 0.030 & 0.002 & $0.912(0.860-0.967)$ \\
Total cholesterol & 0.015 & 0.005 & 0.005 & $1.015(1.005-1.026)$ \\
HDL & -0.018 & 0.016 & 0.263 & $0.982(0.952-1.013)$ \\
Fasting glucose & 0.084 & 0.024 & 0.001 & $1.088(1.037-1.141)$ \\
Fasting insulin & 0.525 & 0.233 & 0.025 & $1.690(1.069-2.670)$ \\
HOMA-IR & 2.181 & 1.019 & 0.032 & $8.853(1.201-65.25)$ \\
HbA1C & 0.721 & 0.514 & 0.160 & $2.057(0.752-5.629)$ \\
\hline
\end{tabular}

SE, standard error; OR, odds ratio; CI, confidence intervals; DBP, diastolic blood pressure; HDL, high-density lipoprotein; HOMA-IR, homeostasis model assessment of the insulin resistance; HbA1C, glycated hemoglobin.

Insulin may improve the bioavailability of insulin like growth factor 1 (IGF-1) by inhibiting the production of IGF-binding proteins. ${ }^{21,22}$ Bioavailable IGF-1 and insulin signal transduction develop pass through insulin, IGF-1, and hybrid receptors in the cell membrane. ${ }^{21,23}$ Inhibition of apoptosis, stimulation of cellular proliferation and carcinogenesis develop because of a number of downstream pathways activated by these receptors. Despite these possible mechanisms, further studies needed to clear the pathophysiological role of hyperglycemia and insulin resistance in gastric carcinogenesis.

This study was able to more accurately survey the effects of insulin resistance in EGC by minimizing confounding factors such as BMI, AC, age, and sex. Identification of risk factors that precede the development of EGC can inform strategies to prevent EGC. Although several studies have evaluated the association between insulin resistance and gastric cancer, this study focused on EGC and possible associations with hyperglycemia and insulin resistance. In a recent study, it was found that there was inverse correlation between serum cholesterol levels and the incidence of gastric cancer, especially intestinal type. ${ }^{23,24}$ It is generally accepted that intestinal type cancer develops by a multistep leading to chronic active gastritis, gastric atrophy process and intestinal metaplasia from normal gastric mucosa and finally to dysplasia and cancer. ${ }^{25}$ These steps include the inflammatory process through activation of the pro-inflammatory transcription factor, nuclear factor $\kappa \mathrm{B}(\mathrm{NF}-\kappa \mathrm{B}){ }^{26}$ Cholesterol is an important structural lipid that required to maintain cell function. ${ }^{27}$ An experimental study showed that long term deficiency of serum cholesterol induces $\mathrm{NF}-\kappa \mathrm{B}$ activation, which could promote tumorigenesis. ${ }^{28}$ Accordingly, we investigated serum lipid profiles related to the development of EGC. Among serum lipid profiles, only TC ( $p=0.005$, $0 R=0.985$ ) was an independent risk factor in our study. In univariate analysis showed that HDL $(p=0.009)$ was statistically significant factor, but not in multivariate analysis. Although cholesterol level is affected by dietary factors and several co-factors, the above mentions would somehow support our results.
Adiponectin is one of the active adipokines involved in the pathogenesis of diabetes through regulation of glucose and fatty acid metabolism and insulin sensitivity in various stromal or epithelial cells. ${ }^{29-31}$ Excess adiposity, including visceral obesity, results in a state of chronic systemic low grade inflammatory state due to the production of inflammatory cytokines by both adipocytes and invasive immune cells, creating a protumorigenic environment. ${ }^{32}$ On the contrary, the circulating level of adiponectin is inversely correlated the risk of some cancers related to body mass index, ${ }^{29}$ and some polymorphisms of adiponectin and its receptor genes are correlated the risk of cancers. ${ }^{33}$ Many studies indicate that adiponectin has anticancer effects and plays an important role in the development and progression of obesity related malignant tumors. ${ }^{33-36}$ Ishikawa et $a .^{37}$ recently reported that low plasma adiponectin concentrations are correlated an increased risk of gastric cancer and adiponectin is potentially involved in the progression of gastric cancer in especially in undifferentiated type cancers of the upper stomach. In a Nakajima et al. ${ }^{38}$ comparing 38 patients with Stage I gastric cancer and controls, adiponectin tended to decrease in cancer patients ( $p=0.0582)$, but the difference was not statistically significant. Based on these previous studies, we investigated the relationship of plasma adiponectin and the development of EGC, excluding AGC. Plasma adiponectin tended to be lower in the EGC group $(8.28 \pm 4.98 \mathrm{ng} / \mathrm{mL})$ than in to control group $(8.61 \pm 6.23 \mathrm{ng} / \mathrm{mL})$, but the difference was not statistically significance. Plasma adiponectin could not be used as a conclusive biomarker for the detection of EGC in our study. However, based on these recent studies low plasma adiponectin levels are correlated an increased risk for gastric cancer and it is likely that adiponectin may play a potential role in cancer progression, ${ }^{39-42}$ further larger scale, well-designed studies are needed to confirm this connection.

This is the first study to examine the relationship of hyperglycemia, insulin resistance, lipid profile and adiponectin in EGC alone, excluding AGC. We revealed that elevated fasting glucose and fasting insulin, HbA1c, HOMA-IR, and serum TC were independent risk factors of the development of EGC. Furthermore, general population with hyperglycemia or other elevated glycemic markers, should be advised to recommend appropriate gastric cancer screenings or surveillance even in the absence of obesity or diabetes.

These are also limitations to this case-control study. First, the average glucose levels were within normal rage in both two group (98.61 vs 87.41). Although EGC group and Control group was not diagnosed overt DM and there was statistical significance, it was not hyperglycemia, not even impaired glucose tolerance of impaired fasting glucose. Although interpretation of results may be a logical leap, but insulin resistance is time consuming and sequential events. As well as obesity and $H$. pylori infection, gastric carcinogenesis appear to be confounded by presence of shared cancer promoting or preventing factors. It 
is difficult to accurate assessment of gastric cancer risk in subjects because of these confounding factors. Thus, investigation into the substantial gastric cancer risk in participants requires adjustment based on these confounding factors. Therefore, welldesigned large scale prospective epidemiological studies are needed to confirm the association between diabetes and gastric carcinogenesis in humans. In conclusion, hyperglycemia and hyperinsulinemia in elevated insulin resistance may be related to the development of EGC.

\section{CONFLICTS OF INTEREST}

No potential conflict of interest relevant to this article was reported.

\section{ACKNOWLEDGEMENTS}

This study was supported by a grant of the Korean Society of Gastrointestinal Cancer for 2012.

Author contributions: H.J.K., M.I.P., and S.K.L. designed study; H.J.K. wrote the paper; S.J.P., W.M., S.E.K., J.H.K, and Y.J.C. reviewed the manuscript; and M.I.P. approved the manuscript.

\section{REFERENCES}

1. Sekikawa A, Fukui H, Maruo T, Tsumura T, Okabe Y, Osaki Y. Diabetes mellitus increases the risk of early gastric cancer development. Eur J Cancer 2014;50:2065-2071.

2. Parkin DM, Bray F, Ferlay J, Pisani P. Global cancer statistics, 2002. CA Cancer J Clin 2005;55:74-108.

3. Park IS, Lee YC, Kim WH, Noh SH, Lee KS, Kim H. Clinicopathologic characteristics of early gastric cancer in Korea. Yonsei Med J 2000;41:607-614.

4. Prabhu SR, Amrapurkar AD, Amrapurkar DN. Role of Helicobacter pylori in gastric carcinoma. Natl Med J India 1995;8:58-60.

5. Kanauchi M. A new index of insulin sensitivity obtained from the oral glucose tolerance test applicable to advanced type 2 diabetes. Diabetes Care 2002;25:1891-1892.

6. Alberti KG, Eckel RH, Grundy SM, et al. Harmonizing the metabolic syndrome: a joint interim statement of the International Diabetes Federation Task Force on Epidemiology and Prevention; National Heart, Lung, and Blood Institute; American Heart Association; World Heart Federation; International Atherosclerosis Society; and International Association for the Study of Obesity. Circulation 2009;120:1640-1645.

7. Harvey AE, Lashinger LM, Hursting SD. The growing challenge of obesity and cancer: an inflammatory issue. Ann N Y Acad Sci 2011;1229:45-52.

8. Hidaka A, Sasazuki S, Goto A, et al. Plasma insulin, C-peptide and blood glucose and the risk of gastric cancer: the Japan Public Health Center-based prospective study. Int J Cancer
2015;136:1402-1410.

9. Shimoyama S. Diabetes mellitus carries a risk of gastric cancer: a meta-analysis. World J Gastroenterol 2013;19:6902-6910.

10. Bråkenhielm E, Veitonmäki N, Cao R, et al. Adiponectin-induced antiangiogenesis and antitumor activity involve caspasemediated endothelial cell apoptosis. Proc Natl Acad Sci U S A 2004;101:2476-2481

11. Motoshima H, Wu X, Mahadev K, Goldstein BJ. Adiponectin suppresses proliferation and superoxide generation and enhances eNOS activity in endothelial cells treated with oxidized LDL. Biochem Biophys Res Commun 2004;315:264-271.

12. Maeda K, Okubo K, Shimomura I, Funahashi T, Matsuzawa Y, Matsubara K. cDNA cloning and expression of a novel adipose specific collagen-like factor, apM1 (AdiPose Most abundant Gene transcript 1). Biochem Biophys Res Commun 1996;221:286-289.

13. Yamauchi T, Kamon J, Ito Y, et al. Cloning of adiponectin receptors that mediate antidiabetic metabolic effects. Nature 2003;423:762-769.

14. Wallace TM, Levy JC, Matthews DR. Use and abuse of HOMA modeling. Diabetes Care 2004;27:1487-1495.

15. Choi ES, Rhee EJ, Kim JH, et al. Insulin sensitivity and insulin secretion determined by homeostasis model assessment and future risk of diabetes mellitus in Korean men. Korean Diabetes J 2008;32:498-505.

16. Ikeda F, Doi Y, Yonemoto K, et al. Hyperglycemia increases risk of gastric cancer posed by Helicobacter pylori infection: a population-based cohort study. Gastroenterology 2009;136:1234-1241.

17. Yamagata H, Kiyohara Y, Nakamura S, et al. Impact of fasting plasma glucose levels on gastric cancer incidence in a general Japanese population: the Hisayama study. Diabetes Care 2005;28:789-794.

18. Dandona P, Thusu K, Cook S, et al. Oxidative damage to DNA in diabetes mellitus. Lancet 1996;347:444-445.

19. Lorenzi M, Montisano DF, Toledo S, Barrieux A. High glucose induces DNA damage in cultured human endothelial cells. J Clin Invest 1986;77:322-325.

20. Yi HK, Hwang PH, Yang DH, Kang CW, Lee DY. Expression of the insulin-like growth factors (IGFs) and the IGF-binding proteins (IGFBPs) in human gastric cancer cells. Eur J Cancer 2001;37:2257-2263.

21. Kasuga M, Ueki K, Tajima N, et al. Report of the Japan diabetes society/Japanese cancer association joint committee on diabetes and cancer. Cancer Sci 2013;104:965-976.

22. Giovannucci E. Insulin and colon cancer. Cancer Causes Control 1995;6:164-179.

23. Asano K, Kubo M, Yonemoto K, et al. Impact of serum total cholesterol on the incidence of gastric cancer in a populationbased prospective study: the Hisayama study. Int J Cancer 2008;122:909-914.

24. Knekt P, Reunanen A, Aromaa A, Heliövaara M, Hakulinen T, Hakama M. Serum cholesterol and risk of cancer in a cohort of 39,000 men and women. J Clin Epidemiol 1988;41:519-530. 
25. Correa P. Human gastric carcinogenesis: a multistep and multifactorial process. First American Cancer Society Award lecture on cancer epidemiology and prevention. Cancer Res 1992;52:67356740.

26. Aggarwal BB. Nuclear factor-kappaB: the enemy within. Cancer Cell 2004;6:203-208.

27. Brown DA, London E. Functions of lipid rafts in biological membranes. Annu Rev Cell Dev Biol 1998;14:111-136.

28. Calleros L, Lasa M, Toro MJ, Chiloeches A. Low cell cholesterol levels increase NFkappaB activity through a p38 MAPK-dependent mechanism. Cell Signal 2006;18:2292-2301.

29. Folsom AR, Kaye SA, Prineas RJ, Potter JD, Gapstur SM, Wallace RB. Increased incidence of carcinoma of the breast associated with abdominal adiposity in postmenopausal women. Am J Epidemiol 1990;131:794-803.

30. Arita Y, Kihara S, Ouchi N, et al. Paradoxical decrease of an adipose-specific protein, adiponectin, in obesity. 1999. Biochem Biophys Res Commun 2012;425:560-564.

31. Díez JJ, Iglesias P. The role of the novel adipocyte-derived hormone adiponectin in human disease. Eur J Endocrinol 2003;148:293-300.

32. Hotta K, Funahashi T, Arita Y, et al. Plasma concentrations of a novel, adipose-specific protein, adiponectin, in type 2 diabetic patients. Arterioscler Thromb Vasc Biol 2000;20:1595-1599.

33. Dal Maso L, Augustin LS, Karalis A, et al. Circulating adiponectin and endometrial cancer risk. J Clin Endocrinol Metab 2004;89:1160-1163.

34. Miyoshi Y, Funahashi T, Kihara S, et al. Association of serum adiponectin levels with breast cancer risk. Clin Cancer Res 2003;9:5699-5704.

35. Goktas S, Yilmaz MI, Caglar K, Sonmez A, Kilic S, Bedir S. Prostate cancer and adiponectin. Urology 2005;65:1168-1172.

36. Michalakis K, Williams CJ, Mitsiades N, et al. Serum adiponectin concentrations and tissue expression of adiponectin receptors are reduced in patients with prostate cancer: a case control study. Cancer Epidemiol Biomarkers Prev 2007;16:308-313.

37. Ishikawa M, Kitayama J, Kazama S, Hiramatsu T, Hatano K, Nagawa H. Plasma adiponectin and gastric cancer. Clin Cancer Res 2005;11(2 Pt 1):466-472.

38. Nakajima TE, Yamada Y, Hamano T, et al. Adipocytokine levels in gastric cancer patients: resistin and visfatin as biomarkers of gastric cancer. J Gastroenterol 2009;44:685-690.

39. Kang JH, Lee YY, Yu BY, et al. Adiponectin induces growth arrest and apoptosis of MDA-MB-231 breast cancer cell. Arch Pharm Res 2005;28:1263-1269.

40. Bub JD, Miyazaki T, Iwamoto Y. Adiponectin as a growth inhibitor in prostate cancer cells. Biochem Biophys Res Commun 2006;340:1158-1166

41. Dieudonne MN, Bussiere M, Dos Santos E, Leneveu MC, Giudicelli Y, Pecquery R. Adiponectin mediates antiproliferative and apoptotic responses in human MCF7 breast cancer cells. Biochem Biophys Res Commun 2006;345:271-279.

42. Ishikawa M, Kitayama J, Yamauchi T, et al. Adiponectin inhibits the growth and peritoneal metastasis of gastric cancer through its specific membrane receptors AdipoR1 and AdipoR2. Cancer Sci 2007;98:1120-1127. 